\title{
Pelvic Splenosis Mimicking Endometriosis; Dysmenorrhea an Endometrioma-like Mass in Sonography
}

\section{A R T I C L E I N F O}

\section{Article Type}

Case Report

\section{Authors}

Saremi A.* $M D$,

Zamanian M. ${ }^{1} M D$

Soltani L. ${ }^{1} M D$

Pooladi A. ${ }^{2} M D, P h D$

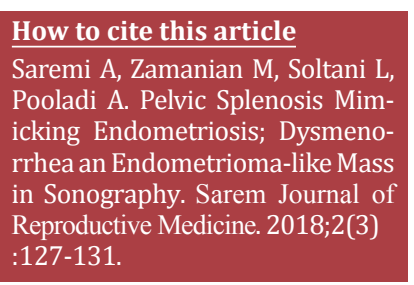

*"Sarem Fertility \& Infertility Research Center (SAFIR)" and "Sarem Cell Research Center (SCRC)", Sarem Women's Hospital, Tehran, Iran ${ }^{1}$ Sarem Women's Hospital, Tehran, Iran

2"Sarem Fertility \& Infertility Research Center (SAFIR)" and "Sarem Cell Research Center (SCRC)", Sarem Women's Hospital, Tehran, Iran

\section{Correspondence}

Address: Sarem Women's Hospital, Basij Square, Phase 3, Ekbatan Town, Tehran, Iran. Postal Code: 1396956111

Phone: +98 (21) 44670888

Fax: +98 (21) 44670432

saremiat@yahoo.com

\section{Article History}

Received: March 23, 2017

Accepted:June 25, 2017

ePublished: August 15, 2018

\begin{abstract}
A B S T R A C T
Patient Information Spontaneous splenic transplantation and implant in in abdominal and pelvic regions is splenosis, which occurs after splenectomy followed by trauma and surgery. The clinical significance of splenic implant and the requirement for its removal are debatable. The cases of splenic implants in the pelvic zone have rarely been reported. Generally, these patients have no clinical features and are usually discovered by chance.

In this report, a 36-year-old woman with the history of splenectomy after an accident at the age of 4 was introduced with Dysmenorrhea and infertility; the primary diagnosis was endometriosis, but pathology results reported the splenosis. Whole tissues and implants of spleen around the ovary and choledosac were removed; following this, the patient's pain was reduced and the overall health improved.

Conclusion Splenosis can emerge as a benign condition in the abdomen and pelvis, and can show itself as a spread of tissue implants in the pelvic cavity. It can, however, easily be mistaken for endometriosis in imaging.
\end{abstract}

Keywords Splenosis; Endometriosis; Ultrasonography; Differential Diagnoses

\section{I T A T I O N L I N KS}

[1] Multiple peritoneal autotransplantation of splenic tissue following traumatic rupture of the ... [2] Splenosis: Multiple peritoneal splenic implants ... [3] Splenosis: A complicating factor in total ... [4] Splenosis: A cause of ... [5] Splenosis: An unrecognized cause ... [6] Splenosis: Report of a case and ... [7] Mayer-Rokitansky-Kuster-Hauser ... [8] Laparoscopic removal of pelvic ... [9] A case of splenosis masquerading ... [10] Portal vein entrance of splenic erythrocytic progenitor cells and local hypoxia of liver, two events cause ... [11] Splenosis: A ... [12] Autotransplantation of spleen ... [13] Thoracic splenosis: A case report and the importance of clinical ... [14] Usefulness of the scintigraphic assessment ... [15] Subcutaneous splenosis: a clue to diagnosis ... [16] Unusual subcutaneous splenosis occurring in a gunshot ... [17] A unique case of cerebral ... [18] Ovarian splenosis: a case ... [19] Splenic neoformation following ... [20] Abdominal ... [21] The histological features of ... [22] Splenosis: The autotransplantation of splenic tissue following injury to the spleen; report of two cases and review of the ... [23] A cause of massive gastrointestinal ... [24] Splenosis presenting as occult gastrointestinal ... [25] Splenosis: Ultrasound and CT findings in a case complicated by an intraperitoneal implant traumatic ... [26] Traumatic rupture of splenic tissue 13 years after splenectomy. A case ... [27] Pelvic splenosis mimicking endometriosis, causing low ... [28] Scintigraphic detection of splenosis causing ureteral compression and ... [29] Idiopathic thrombocytopenic purpura occurring in a subject previously splenectomized for traumatic splenic ... [30] Splenic sequestration of $99 \mathrm{mTc}$ labeled, heat treated red blood ... [31] A case report of abdominal splenosis-a practical mini-review for a ... [32] Splenosis; Review and report of subcutaneous splenic ... [33] Splenogonadal ... [34] Right splenorenal fusion with ... [35] Female splenic-gonadal fusion of the discontinuous ... [36] Thoracic splenosis: A diagnosis by history ... [37] Fine needle aspiration of thoracic splenosis ... [38] Parenchymal thoracic splenosis: History and nuclear imaging without invasive procedures may provide ... [39] Intrathoracic splenosis: Evaluation by $99 \mathrm{mTc}$-labelled heat-denatured erythrocyte [40] MR features of abdominal ... [41] Clinical studies with spleen-specific radiolabeled .. [42] Scintigraphic detection of splenosis: Superiority of tomographic selective spleen ... [43] The born-again spleen. Return of splenic function after splenectomy for ... [44] Minimally invasive surgery in the treatment of ... 


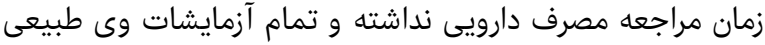

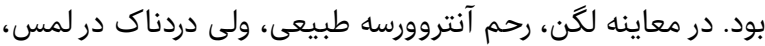

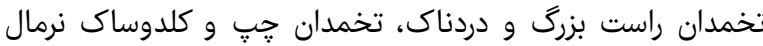

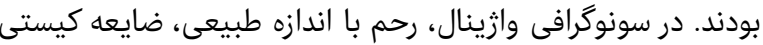

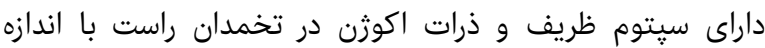

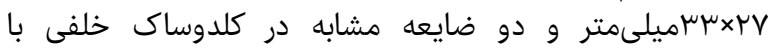

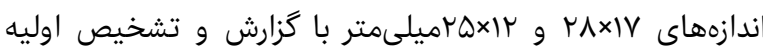

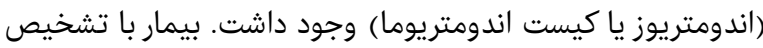

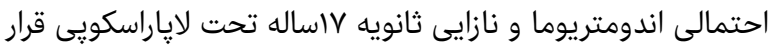

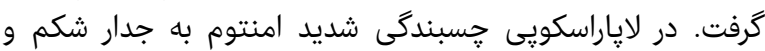

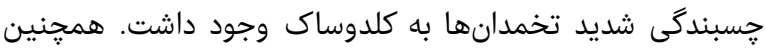

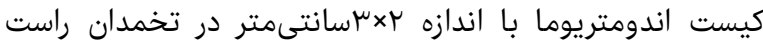

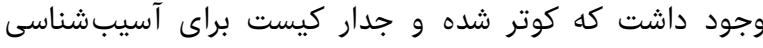

ارسال شد (تصوير ().

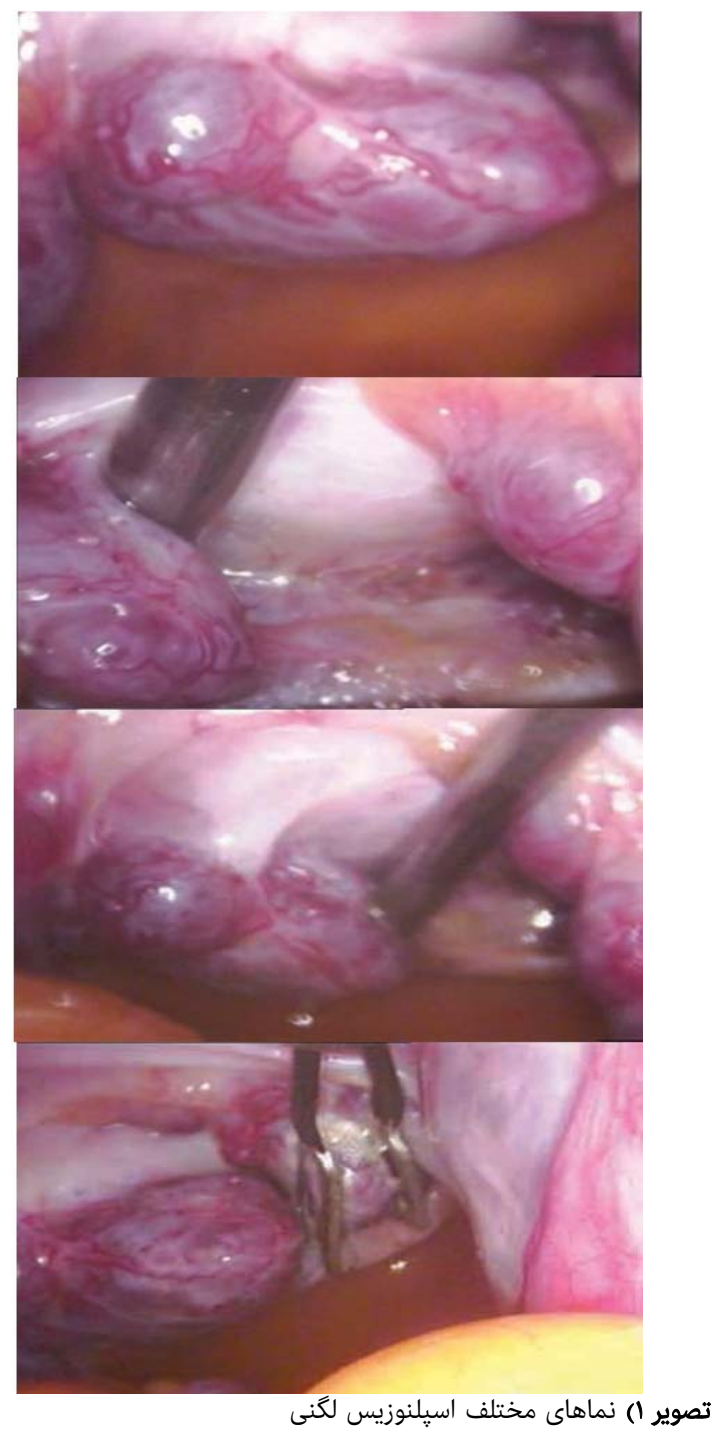

نمونه بيويسى از ضايعات متمايل به رنغ قرمز با با اندازه كمتر از

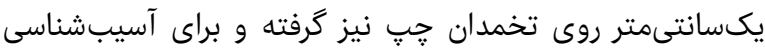

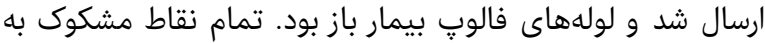

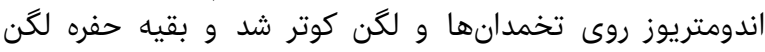

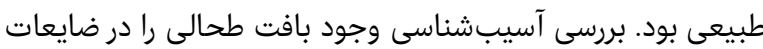

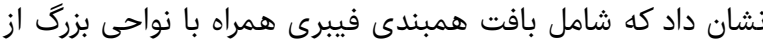

اسيلنوزيس لخنى تقليدكننده اندومتريوز؛ ديسمنوره و توده شبيه اندومتريوما در سونوگرافى لئى

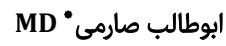

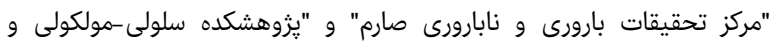

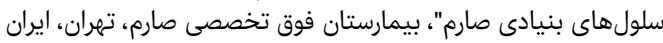

MD مرضيه زمانيان

بيمارستان فوق تخصصى صارم، تهران، ايران

ليلا سلطانى بيمان فيمان

بيمارستان فوق تخصصى صارم، تهران، ايران

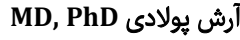

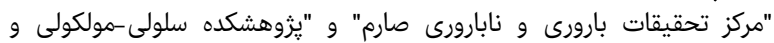

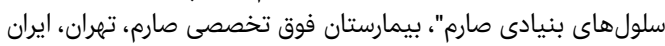

جكيده

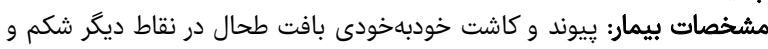

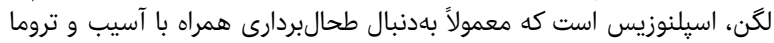

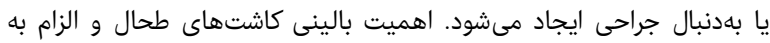

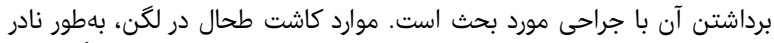

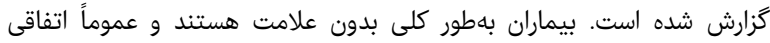

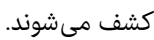

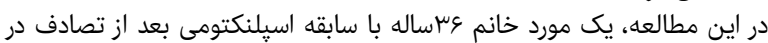

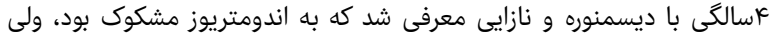

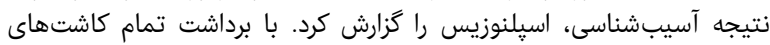

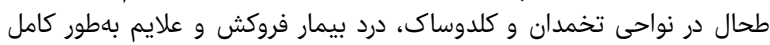

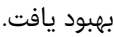

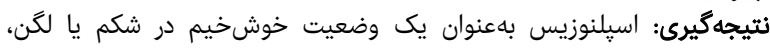

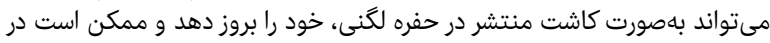

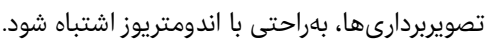

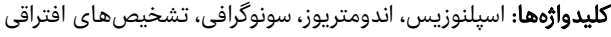

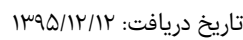

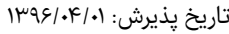
"نويسنده مسئول: saremiat@yahoo.com

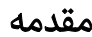

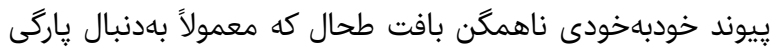

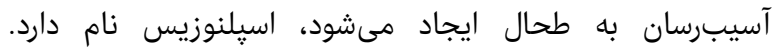

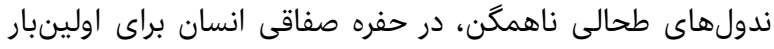

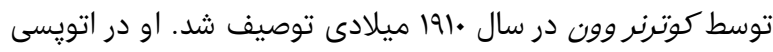

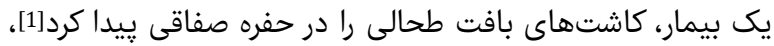

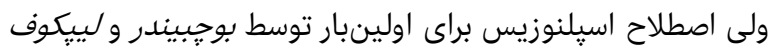

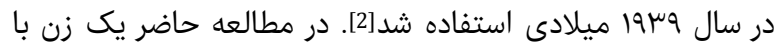

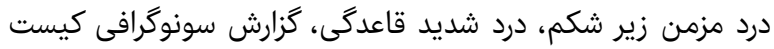

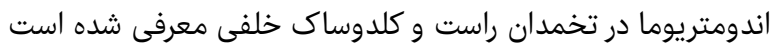
كه در نهايت اسيلنوزيس تشخيص داد راده شد.

\section{بيمار و روشها}

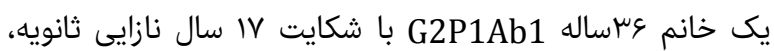

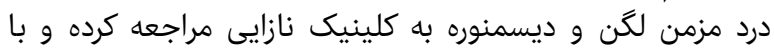

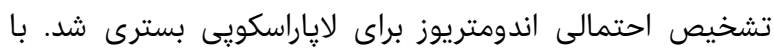

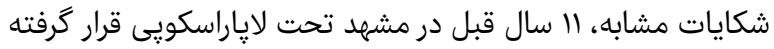

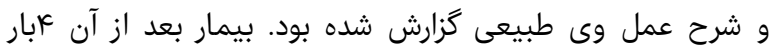

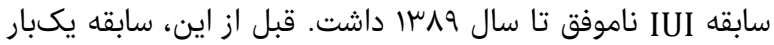
سزارين، كمكارى تيروئيد و كالاكتوره را نيز ذكر كرد كه البته در ائه 


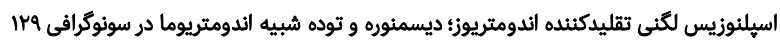

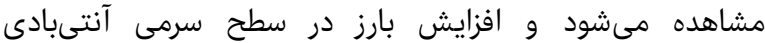

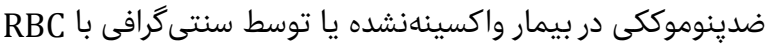

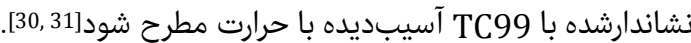

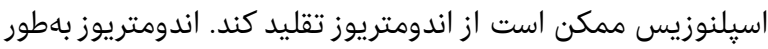

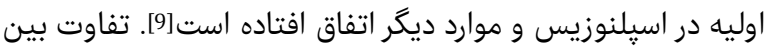

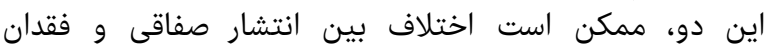

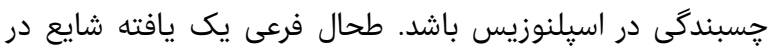

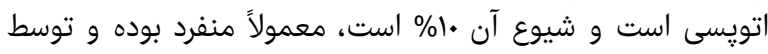

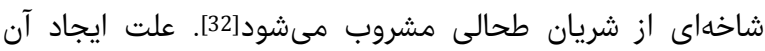

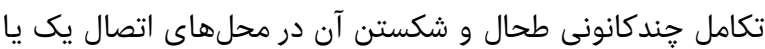

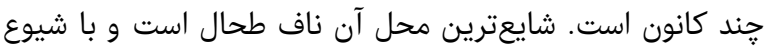

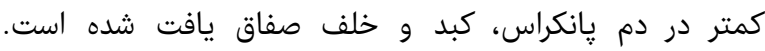

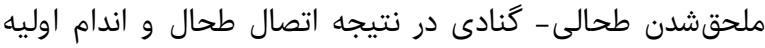

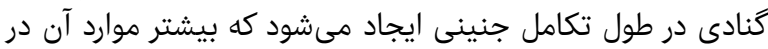

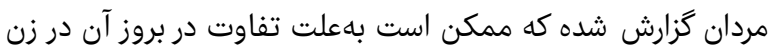

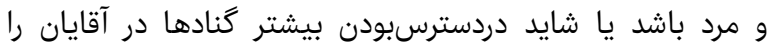

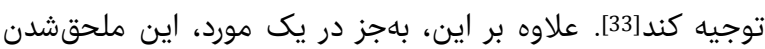

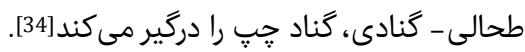

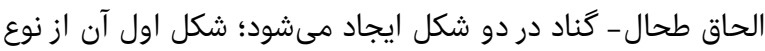

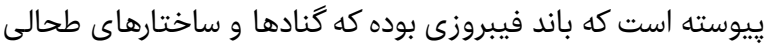

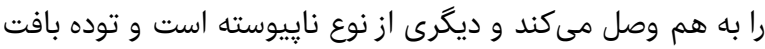

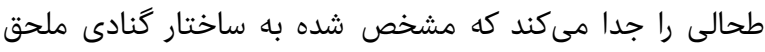

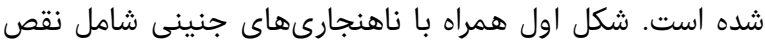

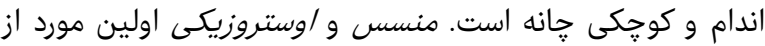

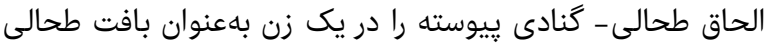

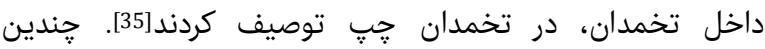

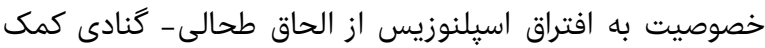

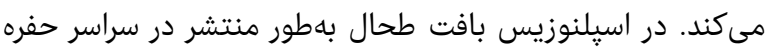

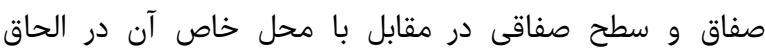

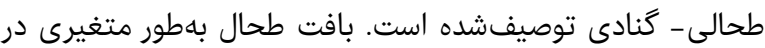

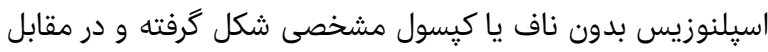

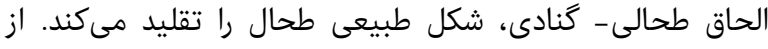

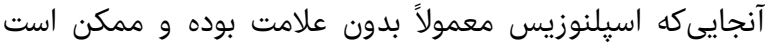

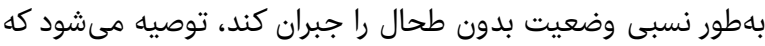

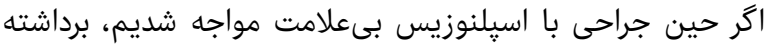

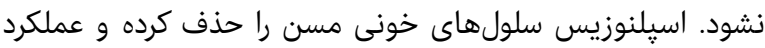

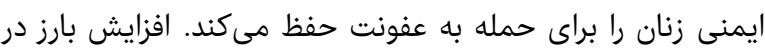

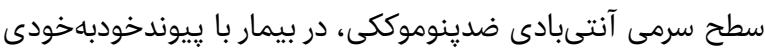

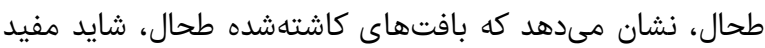

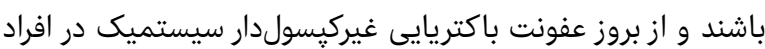

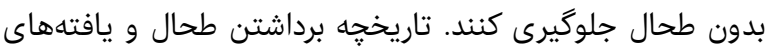

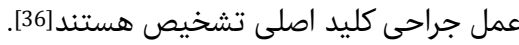

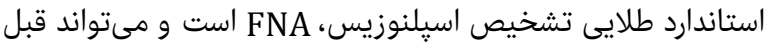

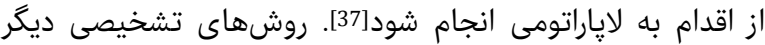

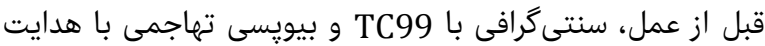

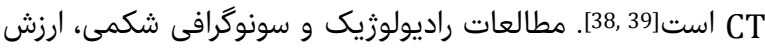

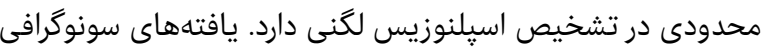

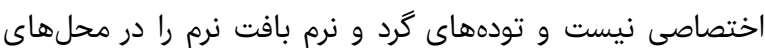

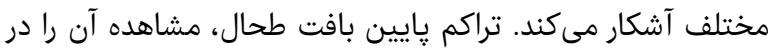

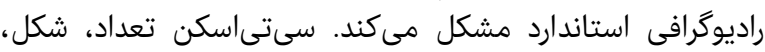

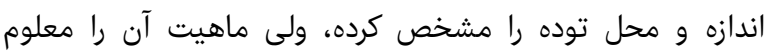

يولي قرمز و سفيد طحال، با نواحى از رسوب يِيگمان هموسيدرين

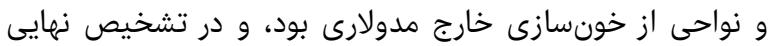

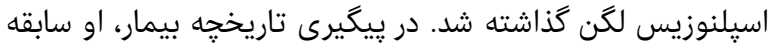

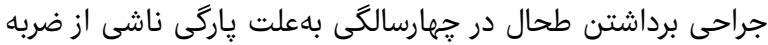

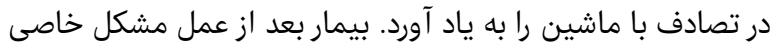

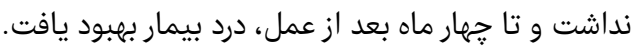

بحث تصادفات جادهاى با ضربه به شكم و عوارض آن، يكى از شايعترين

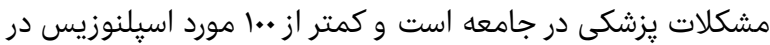

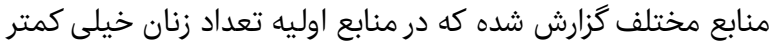

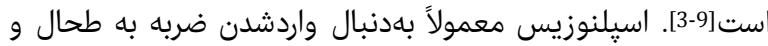

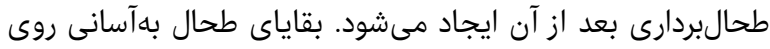

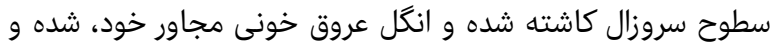

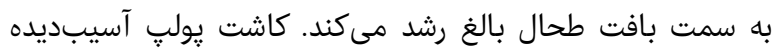

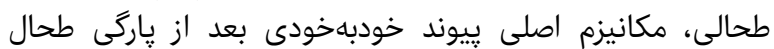

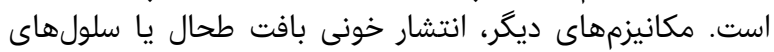

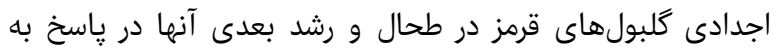

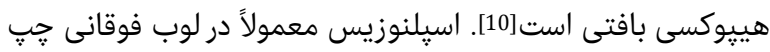

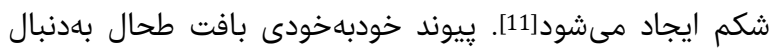

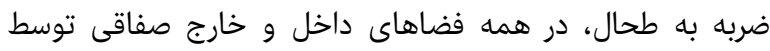

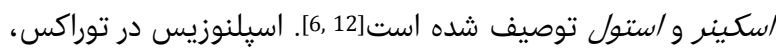

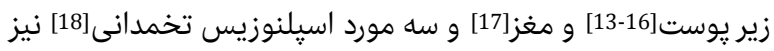

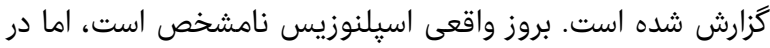

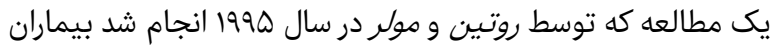

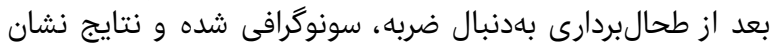

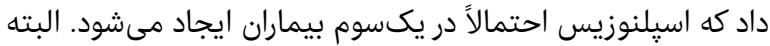

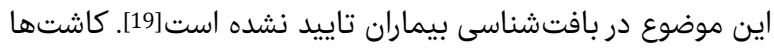

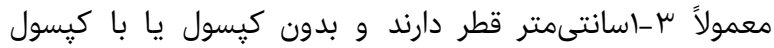

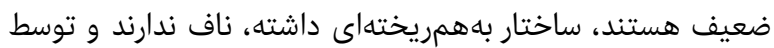

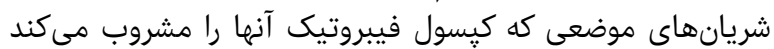

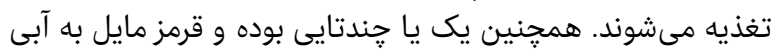

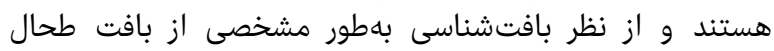

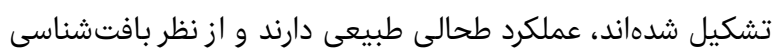

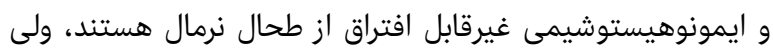

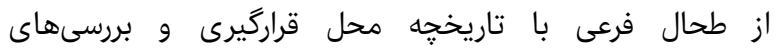
ميكروسكويى قابل افتراق هستند

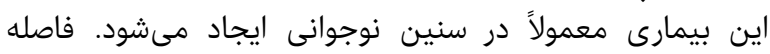

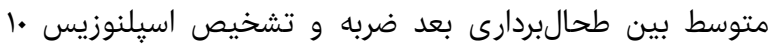

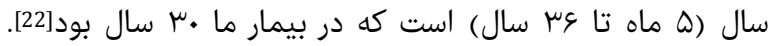

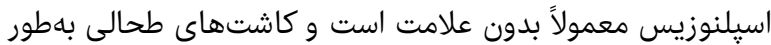

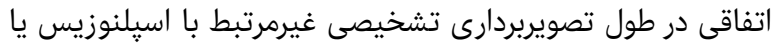

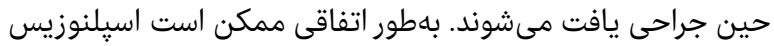

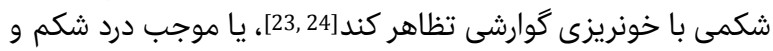

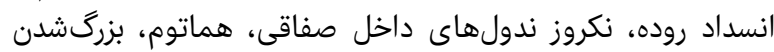

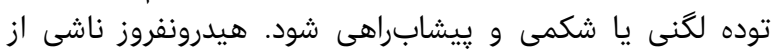

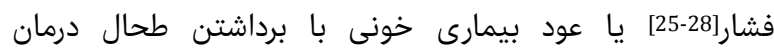

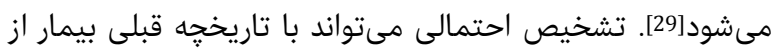

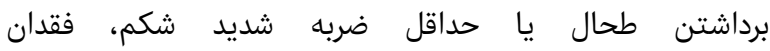

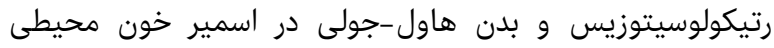

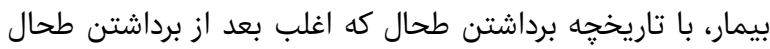


متشكريم. تاييديه اخلاقى: موردى توسط نويسنده گزارش نشده است.

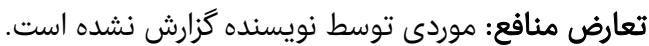

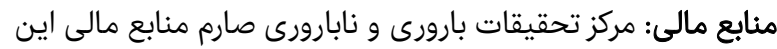
يثزوهش را تامين كرده است.

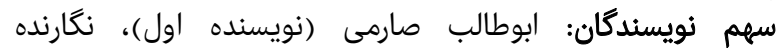

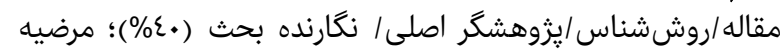

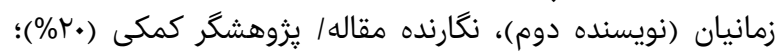

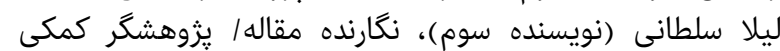

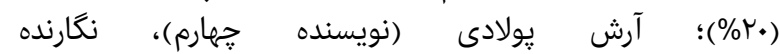

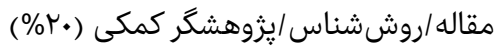

\section{منابع}

1- Waugh Rl. Multiple peritoneal autotransplantation of splenic tissue following traumatic rupture of the Spleen. N Engl J Med. 1946;234:621-5.

2- Buchbinder JH, Lipkoff CJ. Splenosis: Multiple peritoneal splenic implants following abdominal injury. A report of a case and review of the literature. Surgery. 1939;6(6):927-34.

3- Auerbach RD, Kohorn EI, Cornelius EA, Chambers JT. Splenosis: A complicating factor in total abdominal hysterectomy. Obstet gynecol. 1985;65(3 Suppl):65S-8S. 4- Overton TH. Splenosis: A cause of pelvic pain. Am J Obstet Gynecol. 1982;143(8):969-70.

5- Belhassen A, Aubineau JM, Bouverne B, Gautier P. Splenosis: An unrecognized cause of pelvic pain. J Gynecol Obstet Biol Reprod (Paris). 1987;16(3):335-8.

6- Stovall TG, Ling FW. Splenosis: Report of a case and review of the literature. Obstet gynecol surv. 1988;43(2):69-72.

7- Griggs JA, Rudoff J, Coddington CC. Mayer-RokitanskyKuster-Hauser syndrome with splenosis. A case report. J Reprod Med. 1990;35(8):821-3.

8- Higgins RV, Crain JL. Laparoscopic removal of pelvic splenosis. A case report. J Reprod Med. 1995;40(2):1402.

9- Matonis LM, Luciano AA. A case of splenosis masquerading as endometriosis. Am J Obstet Gynecol. 1995;173(3 Pt 1):971-3.

10- Kwok CM, Chen YT, Lin HT, Su CH, Liu YS, Chiu YC. Portal vein entrance of splenic erythrocytic progenitor cells and local hypoxia of liver, two events cause intrahepatic splenosis. Med Hypotheses. 2006;67(6):1330-2.

11- Fremont RD, Rice TW. Splenosis: A review. South Med J. 2007;100(6):589-93.

12- Skinner EF, Hurteau WW. Autotransplantation of spleen into thorax. J Thorac Surg. 1957 Jun;33(6):80713.

13- Kim K, Choi HJ, Kim YM, Kwon WJ, Lee WC, Suh JH. Thoracic splenosis: A case report and the importance of clinical history. J Korean Med Sci. 2010;25(2):299-303

14- Wojcik J, Grodzki T, Kozlowska I, Urbanski S, Kubisa B. Usefulness of the scintigraphic assessment of the thoracic splenosis, a case report. Pneumonol Alergol Pol. 2008;76(6):456-9.

15- Velitchkov NG, Kjossev KT, Losanoff JE, Kavardjikova VA. Subcutaneous splenosis: a clue to diagnosis of thoracic splenosis. J R Coll Surg Edinb. 2000;45(1):66.

16- Yeh CJ, Chuang WY, Kuo TT. Unusual subcutaneous

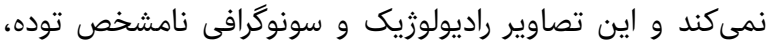

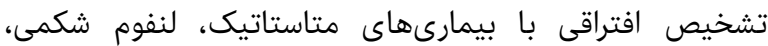

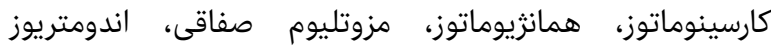

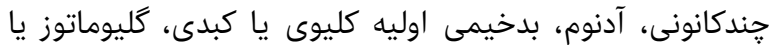

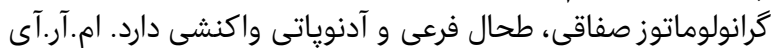
كاشتهاى طحال را با شدت كم در

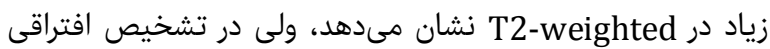

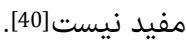
در حال حاضر سنتى مغرافى با TC99 روش استاندارد تشخيصى

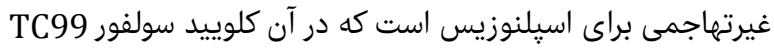

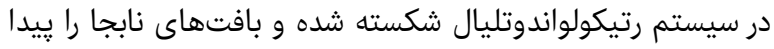

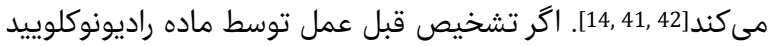

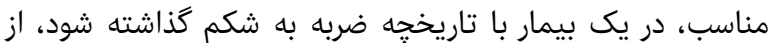

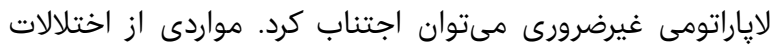

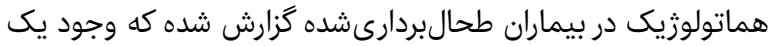

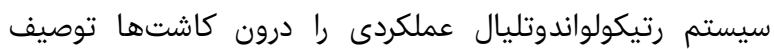

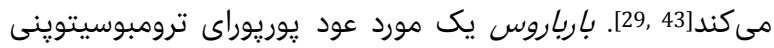

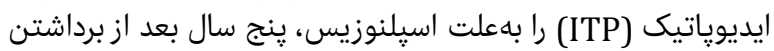

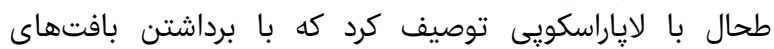

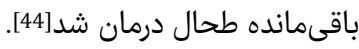
مكانيزم اسيلنوزيس نامشخص استان، ولى إنى استعداد به خونسازى

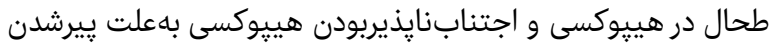

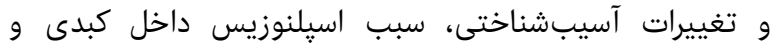

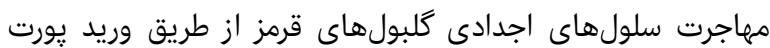

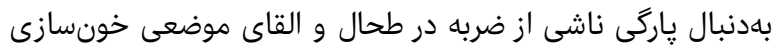

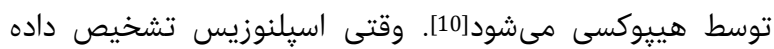

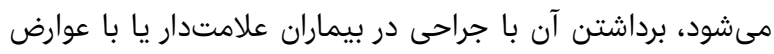

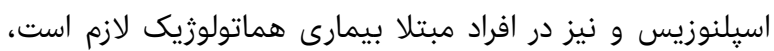

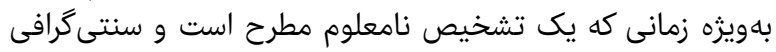

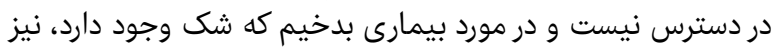
برداشتن جراحى طحال، سودمند است.

$$
\text { نتيجه }
$$

با افزايش شيوع آسيبهاى شكمى بهدليل تصادفات جادهاى و

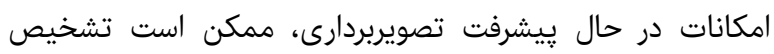

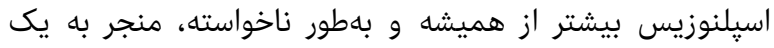

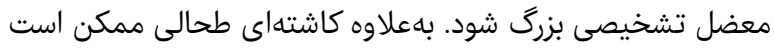

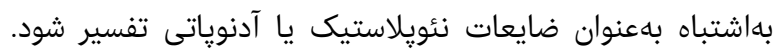

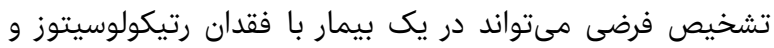

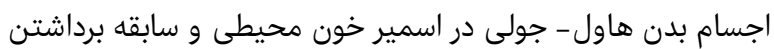

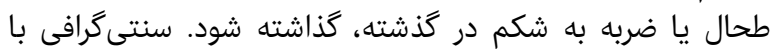
روش غيرتهاجمى قابل اعتماد انتخابى در اين شرايط نادر

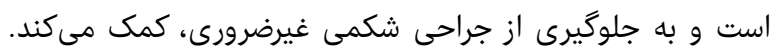

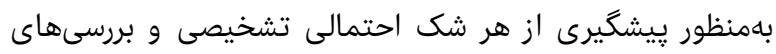

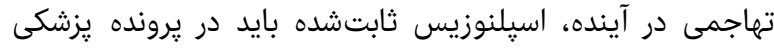
بيمار ثبت شود.

تشكر و قدردانى: از يزشكان و كاركنان بخش نازايى و جراحى

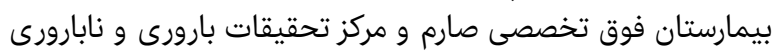

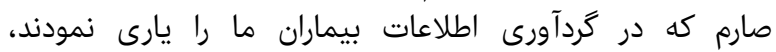


اسيلنوزيس لكنى تقليدكننده اندومتريوز؛ ديسمنوره و توده شبيه اندومتريوما در سونوكرافى اسا 30-Atkins HL, Goldman AG, Fairchild RG, Oster ZH, Som $P$, Richards $P$, et al. Splenic sequestration of $99 \mathrm{mTc}$ labeled, heat treated red blood cells. Radiology. 1980;136(2):501-3.

31- Ksiadzyna D. A case report of abdominal splenosis-a practical mini-review for a gastroenterologist. J Gastrointestin Liver Dis. 2011;20(3):321-4.

32- Cohen EA. Splenosis; Review and report of subcutaneous splenic implant. AMA Arch Surg. 1954;69(6):777-84

33- Watson RJ. Splenogonadal fusion. Surgery. 1968;63(5):853-8.

34- Rosenthal JT, Bedetti CD, Labayen RF, Christy WC, Yakulis R. Right splenorenal fusion with associated hypersplenism J Urol. 1981;126(6):812-4.

35- Meneses MF, Ostrowski ML. Female splenic-gonadal fusion of the discontinuous type. Hum Pathol. 1989;20(5):486-8.

36- Khan AM, Manzoor K, Gordon D, Berman A. Thoracic splenosis: A diagnosis by history and imaging. Respirology. 2008;13(3):481-3.

37- Renne G, Coci A, Biraghi T, Schmid C. Fine needle aspiration of thoracic splenosis: A case report. Acta cytol. 1999;43(3):492-4.

38- Malik UF, Martin MR, Patel R, Mahmoud A. Parenchymal thoracic splenosis: History and nuclear imaging without invasive procedures may provide diagnosis. J Clin Med Res. 2010;2(4):180-4.

39- Crivellaro C, Cabrini G, Gay E, Sara R, Rossetti C. Intrathoracic splenosis: Evaluation by $99 \mathrm{mTc}$-labelled heat-denatured erythrocyte SPECT/CT. Eur J Nucl Med Mol Imaging. 2011;38(2):412.

40- Lin WC, Lee RC, Chiang JH, Wei CJ, Chu LS, Liu RS, et al. MR features of abdominal splenosis. AJR Am J Roentgenol. 2003;180(2):493-6.

41- Armas RR. Clinical studies with spleen-specific radiolabeled agents. Semin Nucl Med. 1985;15(3):26075.

42- Gunes I, Yilmazlar T, Sarikaya I, Akbunar T, Irgil C. Scintigraphic detection of splenosis: Superiority of tomographic selective spleen scintigraphy. Clin Radiol. 1994;49(2):115-7.

43- Pearson HA, Johnston D, Smith KA, Touloukian RJ. The born-again spleen. Return of splenic function after splenectomy for trauma. N Engl J Med. 1978;298(25):1389-92.

44- Barbaros U, Dinccag A, Kabul E. Minimally invasive surgery in the treatment of splenosis. Surg Laparosc Endosc Percutan Tech. 2006;16(3):187-9. splenosis occurring in a gunshot wound scar: Pathology and immunohistochemical identification. Pathol Int 2006;56(6):336-9.

17- Rickert $\mathrm{CH}$, Maasjosthusmann U, Probst-Cousin S, August C, Gullotta F. A unique case of cerebral spleen. Am J Surg Pathol. 1998;22(7):894-6.

18- Talati H, Radhi J. Ovarian splenosis: a case report. Case Rep Med. 2010;2010:472162.

19- Muller U, Rothlin M. Splenic neoformation following trauma-induced splenectomy diagnosis and function. Swiss Surg. 1995;(5):230-5.

20- Pirozynski WJ, Allan CM. Abdominal splenosis. Can Med Assoc J. 1974;111(2):159.

21- Carr NJ, Turk EP. The histological features of splenosis. Histopathology. 1992;21(6):549-53.

22- Cotlar AM, Cerise EJ. Splenosis: The autotransplantation of splenic tissue following injury to the spleen; report of two cases and review of the literature. Annals of surgery. 1959;149(3):402-14.

23- Basile RM, Morales JM, Zupanec R. Splenosis. A cause of massive gastrointestinal hemorrhage. Arch Surg. 1989;124(9):1087-9.

24- Sikov WM, Schiffman FJ, Weaver M, Dyckman J, Shulman R, Torgan P. Splenosis presenting as occult gastrointestinal bleeding. Am J Hematol. 2000;65(1):5661.

25- Delamarre J, Capron JP, Drouard F, Joly JP, Deschepper B, Carton S. Splenosis: Ultrasound and CT findings in a case complicated by an intraperitoneal implant traumatic hematoma. Gastrointest Radiol. 1988;13(3):275-8.

26- Depypere L, Goethals M, Janssen A, Olivier F. Traumatic rupture of splenic tissue 13 years after splenectomy. A case report. Acta chir Belg. 2009;109(4):523-6.

27- Zitzer P, Pansky M, Maymon R, Langer R, Bukovsky I, Golan A. Pelvic splenosis mimicking endometriosis, causing low abdominal mass and pain. Hum Reprod. 1998;13(6):1683-5.

28- Varma DG, Campeau RJ, Kartchner ZA, Karnik S. Scintigraphic detection of splenosis causing ureteral compression and hydronephrosis. AJR Am J Roentgenol. 1991;156(2):406.

29- Mazur EM, Field WW, Cahow CE, Schiffman FJ, Duffy TP, Forget BG. Idiopathic thrombocytopenic purpura occurring in a subject previously splenectomized for traumatic splenic rupture. Role of splenosis in the pathogenesis of thrombocytopenia. Am J Med. 1978;65(5):843-6. 